\title{
Management and Treatment of Paediatric Neurovascular Diseases in an Interdisciplinary Setting
}

Homajoun Maslehaty ${ }^{1 *}$, Athanasios K Petridis ${ }^{2}$, Lutz Schreiber ${ }^{2}$, Friedhelm Brassel ${ }^{3}$, Thorsten Rosenbaum ${ }^{4}$ and Martin Scholz ${ }^{2}$

${ }^{1}$ Department of Neurosurgery, University Hospital Essen, University Duisburg-Essen, Germany

${ }^{2}$ Department of Neurosurgery, Klinikum Duisburg, Sana Kliniken, Academic Teaching Hospital of University Essen-Duisburg, Germany

${ }^{3}$ Department of Radiology and Neuroradiology, Klinikum Duisburg, Sana Kliniken, Academic Teaching Hospital of University Essen-Duisburg, Germany

${ }^{4}$ Department of Pediatrics, Klinikum Duisburg, Sana Kliniken, Academic Teaching Hospital of University Essen-Duisburg, Germany

\begin{abstract}
Introduction: Paediatric neurovascular diseases include different patterns, such as cerebral aneurysms, arteriovenous or vein of Galen and cerebral cavernous malformations, as well as ischemic stroke and other infrequent clinical entities like Moyamoya disease. Management and treatment of this topic requires well-coordinated multidisciplinary care of pediatric neurosurgeons, interventional neuro-radiologists and paediatricians.

Materials and methods: We present six cases of neurovascular pathologies in paediatric patients and highlight the multimodal treatment and management strategy. This case-series included patients with Vein of Galen malformation, Arteriovenous-malformation-bleeding, cerebellar ischaemic stroke, dissecting PICA-aneurysm and one patient with a vascular compression of the vestibular nerve inside the internal auditory meatus.

Results: All patients show a good outcome because of a fast and interdisciplinary work and a close collaboration of the neurosurgeon, the neuroradiologist and the neuropadiatrician.

Discussion: A multimodal therapy-management with an effective interdisciplinary setting including the pediatric neurosurgeon, the interventional neuroradiologist and the neuropaediatricians is necessary to reach the best outcome of the patient. Paediatric neurovascular diseases should be treated in an experienced centre with good infrastructure and good interdisciplinary work.
\end{abstract}

Keywords: Neurovascular emergency; Vein of galen malformation; Arteriovenous malformation; Stroke; Intracranial aneurysm; Pediatric

\section{Introduction}

Paediatric Neurovascular Diseases (PND) is a topic, which includes different patterns, such as cerebral aneurysms, Arteriovenous (AVM) or Vein of Galen (VOG) and Cerebral Cavernous Malformations (CCM), as well as Developmental Venous Anomalies (DVA), ischemic stroke and other infrequent clinical entities like Moyamoya disease.

Although rare in clinical daily routine-compared to cerebrovascular accidents in adults-its treatment and adequate management is of particular importance. Management and treatment of this topic requires well-coordinated multidisciplinary care of neurosurgeons, interventional neuro-radiologists and paediatricians.

In the current short review we present a selection of recently treated cases of PNDs with the special focus on the multidisciplinary management setting.

\section{Case Study}

\section{Case 1: Vein of Galen malformation}

A four months old girl was admitted to the pediatric department of our institution due to a previous diagnosed VOG. Ultrasound through the fontanel was suspicious for a vascular anomaly. Consecutive performed MRI brought the diagnosis of VOG (choroidal type) (Figure $1 \mathrm{a}$ and $\mathrm{lb}$ ). The patient was hospitalized for endovascular embolization of VOG. On admission the girl was alert, without any neurological deterioration with development corresponding to her age.

Digital Subtraction Angiography (DSA) was done and the VOG could be embolized under general anaesthesia completely (Figure 1c). After the procedure the patient was transferred to the pediatric Intensive Care Unit (ICU) and presented no neurological impairments after the recovery phase. However, 12 hours after the procedure, the patient presented with diminished consciousness. The neurosurgeon on duty was consulted immediately and a cranial CT scanning was performed, showing Intraventricular Hemorrhage (IVH) with accompanied occlusive Hydrocephalus (HCP) (Figures 2a-2c). The patient was brought to the Operating Room (OR) and an External Ventricular Drainage (EVD) was implanted right frontal to flush out the blood the control the enlargement of the ventricles.

The clinical condition improved rapidly and control images showed

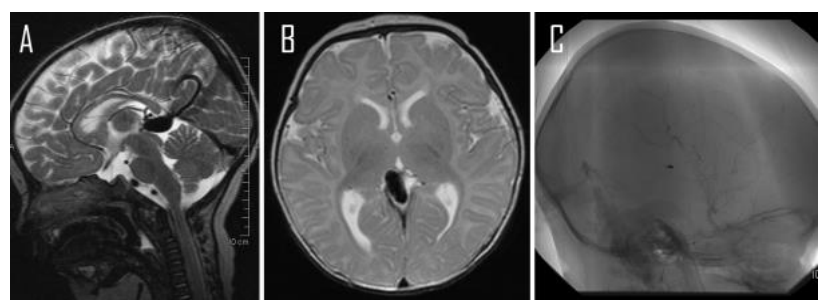

Figure 1: A: Cranial MRI shows choroidal VOG, A: sagittal, B: axial; C: DSA after embolization shows complete occlusion of VOG.

*Corresponding author: Homajoun Maslehaty, Department of Neurosurgery University Hospital Essen, University Duisburg-Essen, Germany, Tel: +49 201 7230; E-mail: h.maslehaty@gmx.de

Received July 01, 2014; Accepted September 22, 2014; Published September 24, 2014

Citation: Maslehaty H, Petridis AK, Schreiber L, Brassel F, Rosenbaum T, et al (2014) Management and Treatment of Paediatric Neurovascular Diseases in an Interdisciplinary Setting. J Neurol Disord 2: 181. doi:10.4172/2329-6895.1000181

Copyright: (c) 2014 Maslehaty H, et al. This is an open-access article distributed under the terms of the Creative Commons Attribution License, which permits unrestricted use, distribution, and reproduction in any medium, provided the original author and source are credited. 


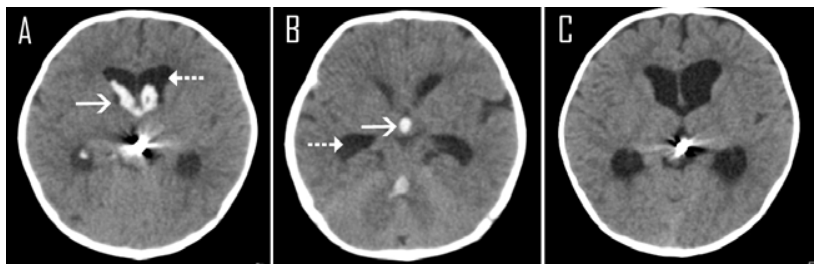

Figure 2: A and B: CT scanning shows hemorrhage in all ventricles with accompanied occlusive hydrocephalus. Arrow: Intraventricularhemorrhage, dotted arrow: enlarged ventricular system; C: CT scanning at time of discharge shows complete resolution of blood.

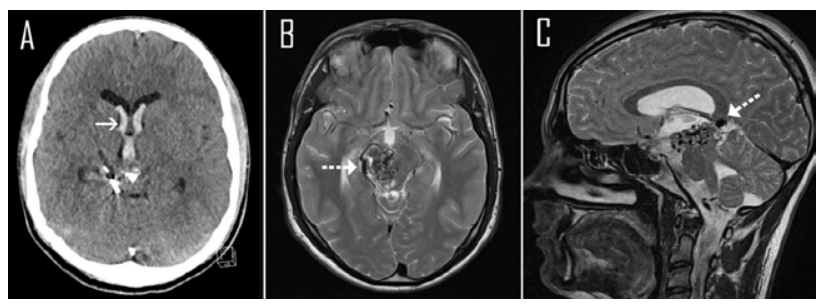

Figure 3 :A: CT scan shows hemorrhage in the brain ventricles and preembolized AVM. Arrow: intraventricularhemorrhage; B and C: axial and sagittal T2-weighted MRI show the extent of the AVM in the brainstem (dotted arrow).

resolution of the blood with normalization of the ventricle size. However, after removal of EVD on day 12 the clinical condition deteriorated once more, with the necessity of implantation of a new EVD. In the continuing course the patient developed meningitis. This was treated sufficiently with antibiotic drugs. The inflammatory factors declined and finally the EVD could be removed, without anew deterioration. The patient was discharged without neurological impairments. On followup (FU) the clinical condition was still stable without occurrence of post infectious HCP.

\section{Case 2: AVM grade 5 within the brainstem}

A 17 years old boy was admitted to our department due to a new occurrence of IVH in line with a previous diagnosed brainstem AVM (Spetzler-Martin grade 5). The AVM was partially embolized three times and treated additionally with gamma-knife radiation during the last few years, which led to a slight decrease of the size and the perfusion of the AVM (Figure 3).

On admission the patient presented with severe headache. However, he was alert and presented no new neurological deterioration, except his pre-existing left sided spasticity, hemiparesis and visual impairments. An EVD was implanted right frontal to control Intracerebral Pressure (ICP) and to obtain IVH drainage. EVD could be removed few days later and the patient was discharged home without new deficits.

One day after discharge the patient was admitted again due to recurrence of IVH. After the interdisciplinary conference on this case we decided to perform once more a partial endovascular embolization with onyx for further reduction of the feeding vessels. After embolization the patient was treated in the Pediatric ICU but didn't woke up properly, so that a cranial MRI was performed, which showed left sided thalamic ischemic stroke (Figure 3). However, his condition improved slowly and he could be discharged finally in a stable clinical condition.

\section{Case 3: AVM grade 2}

A 13 years old girl suffered from an acute onset of severe headache and double vision on her way to school. She was admitted by her family doctor to a regional hospital and was treated conservatively without any neuro-imaging. After strong recommendation for imaging due to progressive vertigo by a called ENT doctor, a cranial CT scan was done, which showed a left parietal $4.5 \mathrm{~cm}$ x $3.5 \mathrm{~cm}$ large hemorrhage (Figure 4a). After diagnosis the patient was transferred to our department for further diagnostic steps and therapy. Additionally performed cranial MRI and DSA showed a left parietal ruptured AVM (Spetzler-Martin grade 2) (Figure $4 \mathrm{~b}$ and $4 \mathrm{c}$ ). After discussion of this case, preoperative embolization of the feeding vessels was done, followed by microsurgical resection of the AVM and removal of hemorrhage.

The patient was treated in the pediatric ICU postoperatively and remained sedated and intubated for further 6 hours for blood pressure control. She recovered well and could be discharged without neurological impairments. Her vertigo and double vision declined completely.

\section{Case 4: Cerebellar ischemic stroke}

An 8 years old boy suffered from head and neck pains since three months with a sudden onset of nausea and vomitus. He was somnolent on admission and presented with horizontal nystagmus. Considering the pre-existing co-morbidities it has to be mentioned that a Kawasakisyndrome was treated sufficiently at the age of three years. Further information of his familiar history showed that two uncles had ischemic strokes in young ages.

Cranial CT scan and MRI showed distinct right sided ischemic stroke of the cerebellum. Angiographic MRI sequences showed absent patency of right sided AICA and PICA (Figure 5). An EVD was placed right frontal for acute treatment of occurred occlusive hydrocephalus. Furthermore we performed microsurgical decompression of the posterior fossa and partial resection of right sided cerebellar tissue.

Postoperatively the patient was treated in the pediatric ICU and was alert. On examination he presented right sided cerebellar ataxia, hypoglossus palsy and dysarthria.

Postoperative DSA showed hypo-plastic basil artery and signs of an elapsed basilar thrombosis. The patient recovered well and as transferred to rehabilitation.

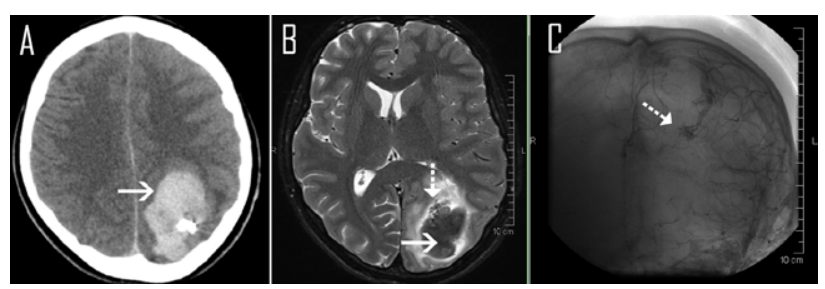

Figure 4: A: CT scan shows ICH (arrow) left parietal after embolization of the feeding vessels; B: T2-weighted MRI shows ICH (arrow) with associated AVM (dotted arrow); C: DSA presentation of AVM (dotted arrow).
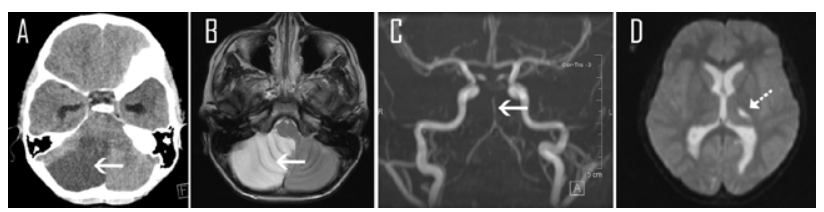

Figure 5: $A$ : CT scan and $B$ : T2-weighted MRI show right cerebellar ischemic stroke (arrow); C: MR-angiography shows hypo-plastic basilar artery (arrow); D: left thalamic ischemic stroke (dotted arrow). 


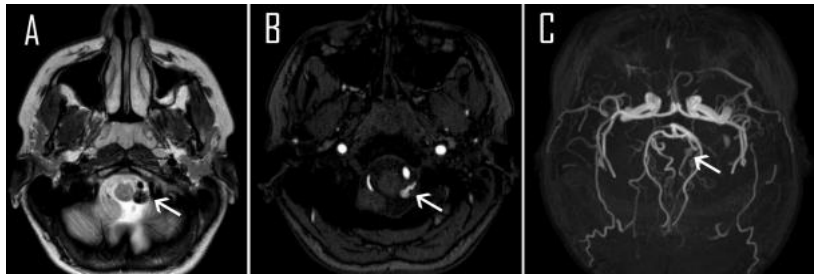

Figure 6: PICA aneurysm (arrows). A: T2-weighted MRI; B: TOF-MRangiography, C: MR-angiography.

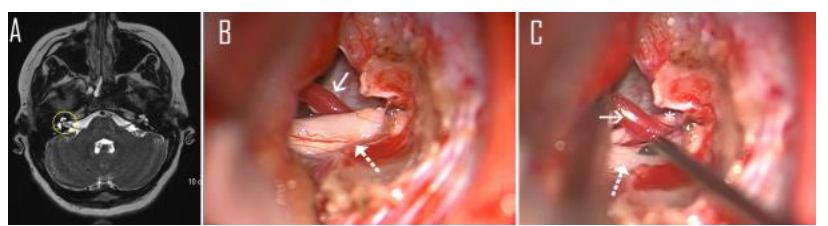

Figure 7: A: T2-weighted MRI (axial) shows neuro-vascular contact (encircled); B\&C: Intraoperative image shows the AICA branch (arrow) runs through the vestibulo-cochlear nerve (dotted arrow) and the facial nerve (asterisk).

\section{Case 5: Dissecting intracranial aneurysm}

A 12 years old girl suffered from progressive left sided headaches with accompanied nausea and photophobia. She was treated with pain killers according to the suspected diagnosis of migraine. After couple of months cranial MRI was performed to rule out other sources. MRI showed a dissecting aneurysm of the Posterior Inferior Cerebellar Artery (PICA) (Figure 6). She was admitted to our institution for further diagnostic evaluation and therapy. The aneurysm was treated with coil embolization and stent implantation. In the continuing course the clinical symptoms declined gradually and the patient could be discharged.

\section{Case 6: Vascular compression of the vestibular nerve}

A 12 years old boy presented with acute onset of severe rotatory vertigo and drop attacks since few days. Performed cranial MRI showed a right sided lesion adjacent to the vestibule-cochlear nerve, assuming the expected diagnosis of a vestibular schwannoma (Figure 7a-7c). Due to the severe clinical symptoms we decide to resect the tumor surgically.

We performed a right sided retrosigmoid craniotomy and identified the vestibulo-cochlear nerve at the internal auditoty meatus. Interstingly, the source of the symptoms was identified as a branch of the Anterior Inferior Cerebellar Artey (AICA) running directly through facial and vestibule-cochlear nerve within the porus accusticus (Figure $7 \mathrm{~b}$, video: supplement material 1). The nerve was decompressed and a muscle graft with fibrin glue was placed between nerve and vessel. The patient recovered well and was finally discharged and experienced significant symptom relief.

\section{Discussion}

Pediatric neurovascular diseases are seen infrequently in clinical daily routine. However, the treatment requires a well-coordinated process in an interdisciplinary setting. A close collaboration of neurosurgeons, interventional neuro-radiologists and neuro-pediatricians in an experienced centre with good infrastructure is essential to obtain appropriate management. In the presented cases we pointed out the necessity of interdisciplinary management and the performed therapy. Subsequently, we give a brief overview of the present literature about management and treatment of each illustrated disease pattern.

VOG malformations are usually treated by endovascular embolization. Transvenous application of ONYX into the malformation is a safe method, which can lead to complete occlusion of these lesions [1]. Complications are rare, but might be severe with a high morbidity and mortality rate. Hence, a close cooperation is important for adequate management of complications [2,3]. In cases of intraventricular hemorrhage transient external ventricular drainage might be necessary for ICP control. Furthermore, permanent shunting in cases of communicating hydrocephalus might be required and should take into account with caution because the patients are adapted to high pressures. The valve pressure adjustments should be done under consideration of this detail.

AVMs are more common vascular anomalies in pediatric patients, which become symptomatic in line with seizures, bleedings, steal syndrome and local mass effect. The treatment become urgently in cases of hemorrhage and status epilepticus. Commonly, these malformations are treated interdisciplinary in two sessions with preoperative embolization of the feeding vessels, followed by surgical removal of the AVM. However, Onyx embolization alone is getting popular. In a series of 25 pediatric patients a complete and partial occlusion of the AVM could be achieved in $12 \%$ and $88 \%$ respectively. The procedure related complication rate is estimated with $5.3 \%$ and the neurological complication rate with $10.5 \%$ [4]. The authors conclude the feasibility of this method for preoperative and primary embolization of AVMs but pointed out the extensive complication rates.

As an adjuvant therapeutically approach after endovascular and surgical treatment of AVMs, stereotactic radiotherapy appears to be a safe and effective method, especially in high grade AVMs. However, radiation induced necrosis with occurrence of seizures and other neurological impairments, as well as asymptomatic imaging variances should be considered exactly $[5,6]$. Due to their relative good results with complete occlusion rates between $64.7 \%$ and $80 \%$ and low morbidity rates during the follow-up, Levy et al. postulate that stereotactic radiosurgery should be the first choice of pediatric AVMs [7].

Kiris et al. came to the conclusion that microsurgical resection of AVMs is still the gold standard for treating these lesions especially in Spetzler-Martin grades 1-3. In their series the authors report the very low morbidity and mortality rates after microsurgery with high obliteration rates of $89 \%$ [8]. Pandey et al. analyzed the multimodal therapy for grade $3 \mathrm{AVMs}$ and came to the conclusion that preoperative endovascular treatment, followed by surgical resection and adjuvant radiotherapy leads to the highest obliteration rates, independent from venous drainage and eloquent location [9].

Ischemic stroke is a very rare clinical entity in the childhood, due to the very low incidence of risk factors of ischemic stroke like arterial hypertension and diabetes mellitus [10]. Ischemic stroke is most frequently caused by cerebral vasculopathies, cardiac disorders, Moyamoya disease, head trauma and infections [11-13]. However, ischemic stroke in children is often overseen and leads to delayed diagnosis, which indicates the need for raising awareness in this clinical entity [10]. The rationale of endovascular treatment is to recanalize the occluded vessel and implantation of an intra-arterial stent to obtain the patency of the affected vessel. The neurosurgical treatment approach is limited and includes decompressive craniectomy with or without resection of the infarcted brain tissue for pressure control.

Intracranial aneurysms are very uncommon in children and this topic is not well studied [14]. Mehritra et al. studied a cohort of 57 
Citation: Maslehaty H, Petridis AK, Schreiber L, Brassel F, Rosenbaum T, et al. (2014) Management and Treatment of Paediatric Neurovascular Diseases in an Interdisciplinary Setting. J Neurol Disord 2: 181. doi:10.4172/2329-6895.1000181

patients with 73 aneurysms, who underwent surgical clipping. The authors conclude that intracranial aneurysms most commonly present with rupture and subarachnoid hemorrhage, followed by paralytic types and aneurysm associated seizures. Furthermore the incidence of giant aneurysms and aneurysms located in the posterior fossa seems to be higher compared to adult patients [14].

The Helsinki group analyzed the data of 59 patients with intracranial aneurysms in the childhood with long term follow-up and clinical and radiologic controls (median 34 years) and conclude that patients with ruptured intracranial aneurysms in the childhood have high risk for new aneurysms and new subarachnoid hemorrhage. The incidence increases further, if the patients start to smoke [15].

Concerning the treatment of intracranial aneurysms, some specialized centres report good results of surgical and other centres again good results of endovascular treatment $[16,17]$. There is similar debate on which treatment is superior compared adult patient groups. Similarly to adult patients, size, shape and localization of the aneurysm are important factors to choose the right method on a case by case basis.

The presented last case is very exceptional. By reviewing the literature, just few cases are reported in which neuro-vascular compression mimic a vestibular schwannoma and cause vertigo [18]. However, the presented case with such a severe vertigo sudden in onset is unique.

\section{Conclusion}

A multimodal therapy-management with an effective interdisciplinary setting including the pediatric neurosurgeon, the interventional neuroradiologist and the neuropaediatricians is necessary to reach the best outcome of the patient. Paediatric neurovascular diseases should be treated in an experienced centre with good infrastructure and good interdisciplinary work.

\section{References}

1. Albuquerque FC, Ducruet AF, Crowley RW, Bristol RE, Ahmed A, et al. (2014) Transvenous to arterial Onyx embolization. J Neurointerv Surg 6: 281-285.

2. Stephan S, Rodesch G, Elolf E, Wiemann D, Jorch G (2012) Vein of galen aneurysmal malformations: an ultrasonographic incidental finding-a case report. Case Rep Pediatr 2012: 824284

3. Ahmad M, Mir S, Wahab S, Rizvi I (2012) Vein of Galen malformation. BMJ Case Rep 2012.
4. Soltanolkotabi M, Schoeneman SE, Alden TD, Hurley MC, et al. (2013) Onyx embolization of intracranial arteriovenous malformations in pediatric patients. $J$ Neurosurg Pediatr 11: 431-437.

5. Blamek S, Larysz D, Miszczyk L (2013) Stereotactic linac radiosurgery and hypofractionated stereotactic radiotherapy for pediatric arteriovenous malformations of the brain: experiences of a single institution. Childs Nerv Syst 29: 651-656.

6. Darsaut TE, Guzman R, Marcellus ML, Edwards MS, Tian L, et al. (2011) Management of pediatric intracranial arteriovenous malformations: experience with multimodality therapy. Neurosurgery 69: 540-556.

7. Levy El, Niranjan A, Thompson TP, Scarrow AM, Kondziolka D, et al (2000) Radiosurgery for childhood intracranial arteriovenous malformations. Neurosurgery 47: 834-841.

8. KiriÄŸ T, Sencer A, SahinbaÅ̈̈ M, Sencer S, Imer M, et al. (2005) Surgical results in pediatric Spetzler-Martin grades I-III intracranial arteriovenous malformations. Childs Nerv Syst 21: 69-74.

9. Pandey P1, Marks MP, Harraher CD, Westbroek EM, Chang SD, et al (2012) Multimodality management of Spetzler-Martin Grade III arteriovenous malformations. J Neurosurg 116: 1279-1288.

10. Jordan LC, Hillis AE (2011) Challenges in the diagnosis and treatment of pediatric stroke. Nat Rev Neurol 7: 199-208.

11. Xie LL, Jiang L (2013) Arterial ischemic stroke and hemorrhagic stroke in Chinese children: A retrospective analysis. Brain Dev.

12. Lagman-Bartolome AM, Pontigon AM, Moharir M, MacGregor DL, Askalan R et al. (2013) Basilar artery strokes in children: good outcomes with conservative medical treatment. Dev Med Child Neurol 55: 434-439.

13. Sfaihi L, Elloumi S, Fourati H, Kamoun T, Mnif Z, et al. (2013) Arterial ischemic stroke in children: 22 cases from southern Tunisia. Fetal Pediatr Pathol 32: 271-275.

14. Mehrotra A, Nair AP, Das KK, Srivastava A, Sahu RN, et al. (2012) Clinical and radiological profiles and outcomes in pediatric patients with intracranial aneurysms. J Neurosurg Pediatr 10: 340-346.

15. Koroknay-Pál P, Niemelä M, Lehto H, Kivisaari R, Numminen J, et al. (2013) De novo and recurrent aneurysms in pediatric patients with cerebral aneurysms. Stroke 44: 1436-1439.

16. Saraf R, Shrivastava M, Siddhartha W, Limaye U (2012) Intracranial pediatric aneurysms: endovascular treatment and its outcome. J Neurosurg Pediatr 10: 230-240.

17. Fulkerson DH, Voorhies JM, Payner TD, Leipzig TJ, Horner TG, et al. (2011) Middle cerebral artery aneurysms in children: case series and review. J Neurosurg Pediatr 8: 79-89.

18. Wuertenberger CJ, Rosahl SK (2009) Vertigo and tinnitus caused by vascular compression of the vestibulocochlear nerve, not intracanalicular vestibula schwannoma: review and case presentation. Skull Base 19: 417-424. 